\title{
A Case Study of a Systematic Iterative Design Methodology and its Application in Engineering Education
}

\author{
Anson Wong \\ Ph.D. Candidate \\ Dept. of Mechanical \& Industrial Engineering \\ University of Toronto, Toronto, Ontario \\ awong@mie.utoronto.ca
}

\begin{abstract}
Iterative design is a design methodology based on a cyclical process of idea generation, evaluation, and design improvement until the design requirement is met. It is a widely used design strategy due to its intuitive nature and effectiveness in facilitating design improvement. As technologies advance rapidly nowadays, the level of complexity of design problems also increases. It is often impossible to develop a good design solution in the first attempt, making the effective application of iterative design strategy even more important. Over the years, researches have been carried out to refine the iterative design strategies and attempts have been made to integrate these design strategies into engineering education. Nevertheless, more efforts should be expended in the field of engineering education to encourage effective use of iterative design strategies by both educators and students. To this end, this paper presents a case study on the development of a plastic foaming visualization system to demonstrate the effectiveness of the axiomatic design approach, which is a systematic iterative design methodology. The methodology is based on two previously established design axioms that were designed to guide idea generation, as well as to streamline analysis and evaluation processes in a product development cycle. In addition, this paper explores possible methods to improve students' learning experiences by integrating iterative design elements in engineering education with regards to course design and assessment/evaluation tools.
\end{abstract}

\section{Introduction}

Design is a cornerstone for the engineering profession. Until recent decades, the engineering curricula across North America were predominantly scientifically-oriented, while education and practices on design were lacking in the curricula. As a result, there was an increase in dissatisfaction among engineering industries regarding the deficiencies in design ability of engineering graduates. In the late

\author{
Chul B. Park \\ Professor \\ Dept. Mechanical \& Industrial Engineering \\ University of Toronto, Toronto, Ontario \\ park@mie.utoronto.ca
}

1980s, engineering industries and academia recognized the need to made significant changes to engineering curricula [1][2]. This statement was echoed in the 1991-1992 Accreditation Board of Engineering and Technology (ABET) President's Report from Prados [3]. Similarly, a survey pinpointed the lack of design skills as one of the top weaknesses in Canadian engineering curricula [4]. In this context, there has been a growing emphasis to improve learning and assessment of engineering design. This is evident in the increased collaborative efforts by federal funding agencies, industries and accrediting bodies to incorporate design elements in engineering curricula, such as, engineering praxis and capstone courses [5][6][7]. Also, ABET now requires engineering programs to identify, assess, and demonstrate evidence of design competency [8]. A similar accreditation requirement is also incorporated in the Canadian Engineering Accreditation Board [9].

As technology advances rapidly, the level of complexity in design problems also increases. Moreover, engineering design problems are often ambiguous with multiple solutions [10]. In such cases, linear problem solving methodology has shown to be insufficient [11][12]. Rather, designs for these problems are often carried out with an iterative design strategy - a cyclical and iterative process of idea generation, evaluation, and design improvement under which the design requirement is met [13][14][15][16]. During an iterative design process, the understanding of the problem evolves as designers gather and filter more information during the stage of generating and evaluating possible solutions. New constraints, ambiguities and contradictions are often uncovered in such activities, which can lead to a revised problem scoping. Hence, new and often better solutions results. In this context, it is important for engineering students to learn and practice effective use of iterative design, so they will be well-equipped to solve complex, illstructured engineering problems in the future.

In order to improve students' design knowledge and skills, iterative design methodologies should be integrated effectively into various aspects of engineering curriculum, such as course design and 
student assessments [17]. A number of studies have been conducted to provide suggestions to improve design educations. For example, Danielson and Danielson [18] suggested to provide instructions early in problem solving strategies to prevent misconceptions in design activities. Cox [19] proposed to expose students to a broad range of design problems: starting from poor-defined problems and progressing to ill-defined complex problems. Devon and Dorricott [20] advocated that design projects should follow a thorough iterative process that incorporates assessment from multiple perspectives.

In a large-scale study conducted by Atman et al. [21], it was observed that first year undergraduate students (i.e., freshmen) did not practice iterative design as effectively as senior undergraduate students due to the lack of knowledge and design experience. In particular, it was found that the seniors iterate more in the design process and are quicker at it also. Although this suggests that the students improved over the journey of their undergraduate studies, more help should be directed to the freshmen to accelerate their learning curves, so they can become better designers as seniors and later as engineers. In this context, an easy-to-follow, systematic iterative design methodology will be beneficial to the development of engineering students. For example, Radcliffe \& Lee [22] found that the use of a systematic and iterative design methodology correlated positively with the efficiency of the design process and the quality of the product. An example of a systematic iterative design strategy is called the axiomatic design method [23][24]. The methodology is based on two previously established design axioms (i.e., independence axiom and information axiom) that were designed to guide idea generation, as well as to streamline analysis and evaluation processes in product or process development. A design is deemed to be a good one when it satisfies both design axioms. This paper presents a case study on the development of a novel plastic foaming visualization system to demonstrate the effectiveness of the axiomatic design methodology. This paper also explores possible methods to improve students' learning experiences by integrating the concept of iterative design in course design and assessment/evaluation tools.

\section{Axiomatic Design}

Although it is not the main purpose of this paper to detail the principles and concepts of the axiomatic design approach, some key concepts are presented in the following to help readers understand the case study given in Section 3.

\subsection{Key Concepts of Axiomatic Design [23][24]}

The axiomatic design methodology is a systematic iterative design technique for product or process design. It encompasses the whole development cycle: from problem definition, to idea generation, to analysis and evaluation. Using this methodology, designers first identify customers' needs and map them onto a list of high-level functional requirements (FRs) (i.e., what we want to achieve) in the functional domain. These FRs will then be mapped onto the physical domain as a set of design parameters (DPs) (i.e., how we choose to achieve what we want) once a design idea is generated. During the mapping process between FRs and DPs, two axioms must be satisfied:

Axiom 1: The Independence Axiom - Maintain the independence of functional requirements.

Axiom 2: The Information Axiom - Minimize the information content.

To check if axiom 1 is satisfied, the mapping process of FRs and DPs are modelled by a design equation:

$$
\{F R s\}=[A]\{D P s\}
$$

An element of the design matrix, $A_{i j}$, denote the sensitivity of a change in $F R_{i}$ due to a change in $D P_{j}$ :

$$
A_{i j}=\frac{\partial F R_{i}}{\partial D P_{j}}
$$

If the design matrix $\mathrm{A}$ can be made into a diagonal matrix or a triangular matrix, then Axiom 1 is satisfied, and the resulting design is denoted as uncoupled design or decoupled design, respectively. Otherwise, the design is a coupled one and redesigning is needed. Also, FRs are often revised iteratively as the understanding of the design problem evolved during the design process. Axiom 2 can be used to select and/or refine the best solution among those satisfying Axiom 1. According to Axiom 2, the best solution is the one that minimize the information content: the design solution that is simplest and has the highest probability of achieving the FRs.

For more complex problems, the FRs and DPs can be decomposed into a hierarchy. The decomposition of each domain must depend on the hierarchy of the other domain. For example, in order to proceed and decompose a given level of FRs, the DPs in the same level must be established and satisfy Axiom 1 . Then, the decomposed FRs can be identified using the higher level DPs as constraints. This zigzagging procedure can be repeated until the design solution is detailed enough to be executed.

\subsection{Why Axiomatic Design?}

Axiomatic design presents a unified approach that encompasses the entire design cycle in product 
and process development. It has a well-defined and systematic iterative procedure with analysis and assessment tools based on objective measures. In particular, one major step in axiomatic design is the identification and revision of FRs, which oblige designers to translate abstract customers' needs into quantifiable objectives (FRs) and make revisions as needed. This often leads to good problem scoping practices, which can translate into better performance for the final product [25]. In the process of defining and refining appropriate FRs and DPs, designers are often engaged in frequent self-explanation, which helps designers to elaborate their understanding of the problem and solutions, as well as the subsequent improvement in the solutions [26][27]. Also, Axiom 1 allows designers to eliminate unsuitable solutions early and quickly. Meanwhile, Axiom 2 helps to select and refine the best solution among feasible ones, using quantifiable and objective measures. The objectivity in idea selection helps to eliminated socially mediated iterations (e.g., selection of ideas based on negotiation with persuasive tactics, and disruptions in design activities due to difference in opinions and miscommunications), which were shown to be detrimental to the performance of the final product [28]. These tools allow even freshmen to select good refinement in early stages of the design process, which is indicative of good design practice [29]. It also forces designers to consider more constraints (e.g., manufacturability of the product), as well as performance indicators (e.g., quantification of the probability of achieving the FRs), which is known to improve designs [30].

Dwarakanath and Wallace [31] found that designers tend to simultaneously generate and evaluate design solutions as well as modify design criteria. In such processes, designers tend to forget some of the previously defined criteria and may repeat earlier assessments. This problem can be eliminated when the axiomatic design methodology is adopted because designers are required to define and keep track of the hierarchy of FRs and DPs and the mapping between them throughout the design process. Also, decomposition of FRs and DPs in a systematic hierarchy allows designers to put any module on hold and work on another related one. As modules are revisited, they are instantiated in a different context and in the process the designers may recognize new insight [32]. Moreover, the iterative process of identification and decomposition of FRs and DPs allow designers to consider new requirements that cannot be identified or studied until part of the system has been designed. This process is similar to the "reflection-in-action" process that Schon [33] believed to be integral to design.

\section{Case Study}

\subsection{Description of the Perceived Needs}

Foamed plastics with high cell densities and uniform cell size distributions offer superior mechanical properties such as higher toughness and specific tensile stress, as well as better thermal and acoustic insulation properties, when compared to their solid counterparts [34][35][36]. Plastics foaming is a complicated process that involves various thermodynamic, kinetic and mass transfer phenomena. Once molten plastics is saturated with blowing agents, introducing a thermodynamic instability such as a rapid pressure drop to the plastic-gas mixture could initiate bubbles formation and growth in the mixture. The foamed plastic will then be stabilized by cooling. During the foaming process, the plastic-gas mixture may experience a variety level of stresses inside the foam processing equipments, such as, extrusion or injection molding equipments. Previous researches showed that these stresses will induce cell nucleation and affect cell growth [37][38][39], but the foaming mechanisms are still not thoroughly understood. To fill this gap, a device that allows for the in situ visualization of the foaming behaviours of polymer has been developed recently. Axiomatic design methodology was used in the iterative design process of this system. Due to the complexity of the system, only selected sections of the design process is presented in this paper for illustration purpose.

\subsection{Design Process}

From the perceived needs, the major functional requirement and design parameter were identified:

$\mathrm{FR}_{\mathrm{M}}=$ study plastic foaming with extension stress

$\mathrm{DP}_{\mathrm{M}}=$ a system to in situ visualize and capture

plastic foaming process with extension stress

The first level FRs are created with $\mathrm{DP}_{\mathrm{M}}$ as constraint:

$$
\begin{aligned}
& \mathrm{FR}_{1}=\text { apply extensional stress on a plastic sample } \\
& \mathrm{FR}_{2}=\text { foam the plastic sample } \\
& \mathrm{FR}_{4}=\text { capture foaming in situ }
\end{aligned}
$$

A preliminary design to apply extensional stress to a plastic sample was generated based on a stationary and orbiting roller mechanism, which is a common design of extensional rheometer (see Figure 1). Then, the first level DPs were selected accordingly to satisfy each of the 1st level FRs, and the design was analyzed.

$$
\begin{aligned}
& \mathrm{DP}_{1}=\text { a stationary and orbiting roller mechanism } \\
& \mathrm{DP}_{2}=\text { a plastic foaming system with visible } \\
& \text { compartment }
\end{aligned}
$$


$\mathrm{DP}_{3}=$ an high speed camera with a zoom lens

$$
\left[\begin{array}{l}
F R_{1} \\
F R_{2} \\
F R_{3}
\end{array}\right]=\left[\begin{array}{lll}
x & x & o \\
x & x & o \\
x & x & x
\end{array}\right]\left[\begin{array}{c}
D P_{1} \\
D P_{2} \\
D P_{3}
\end{array}\right]
$$

In the design matrix, the $x$ 's and $o$ 's represent strong and no/weak relationships, respectively. $\mathrm{FR}_{1}$ could be achieved primarily by the stationary and orbiting roller system, but the design of the foaming chamber, which is a component of the foaming system $\left(\mathrm{DP}_{2}\right)$, also restricted the range of motion of the rollers. $\mathrm{FR}_{2}$ is depended on both $\mathrm{DP}_{1}$ and $\mathrm{DP}_{2}$ because the stresses applied and the operating conditions (e.g., temperature and pressure) affects the foaming behaviors. Finally, $\mathrm{FR}_{3}$ is achieved by the high speed camera with zoom lens, but the position of the rollers mechanism and the chamber design also impact the visibility to the plastic sample. From this analysis, it was shown that this design was a coupled design since the design matrix was not a diagonal/triangular matrix, and redesign was needed.

Figure 1 . The stationary \& orbiting rollers mechanism

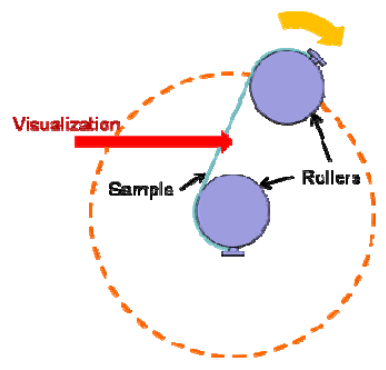

In the revised design, a stationary and orbiting roller mechanism was replaced with a counter-rotating rollers mechanism for the application of extensional stress on a polymer sample $\left(\mathrm{DP}_{1}\right)$ (see Figure 2 ), while all the other FRs and DPs remained unchanged. The FRs-DPs relationships are similar to the previous design except that $\mathrm{FR}_{1}$ was no longer depended on the chamber design because the locations of the rollers were stationary during operation. $\mathrm{FR}_{3}$ also became independent of $\mathrm{DP}_{1}$ because the rollers could be positioned away from the optical axis of the camera. The overall FRs-DPs relationship is summarized in the following:

$$
\left[\begin{array}{l}
F R_{1} \\
F R_{2} \\
F R_{3}
\end{array}\right]=\left[\begin{array}{lll}
x & o & o \\
x & x & o \\
o & x & x
\end{array}\right]\left[\begin{array}{l}
D P_{1} \\
D P_{2} \\
D P_{3}
\end{array}\right]
$$

This foaming visualization system is a decoupled design since the design matrix is a triangular matrix, and hence Axiom 1 was satisfied. Due to the complexity of the system, decomposition of FRs and DPs were needed. In particular, the decomposition process of $\mathrm{FR}_{2}$ and the corresponding DPs is presented in the following.
Figure 2. The counter-rotating rollers mechanism

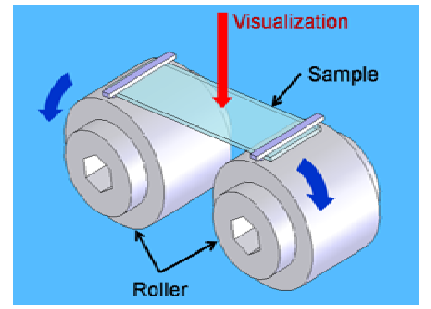

The primary $\mathrm{DP}$ for $\mathrm{FR}_{2}$ is a plastic foaming system with a visible compartment. A supply of high pressure gas to a foaming chamber is needed allow the dissolution of gas into the plastic sample at a desired pressure. The system must also be able to control the foaming temperature, and to release the system pressure at a desired rate to initiate foaming. Based on these requirements, and $\mathrm{DP}_{2}$ as a constraint, the lower level FRs were developed:

$\mathrm{FR}_{21}=$ supply gas at the desired pressure

$\mathrm{FR}_{22}=$ control the system temperature

$\mathrm{FR}_{23}=$ allow for gas saturation without obstructing

the view to the plastic sample

$\mathrm{FR}_{24}=$ release gas at a desired pressure drop rate

The corresponding DPs were selected as:

$\mathrm{DP}_{21}=$ a syringe pump connected to a gas cylinder

$\mathrm{DP}_{22}=$ a temperature controlling system

$\mathrm{DP}_{23}=$ a high-pressure chamber with visible

compartment

$\mathrm{DP}_{24}=$ an adjustable gas release valve

$\mathrm{FR}_{21}$ can be achieved by $\mathrm{DP}_{21}$ because a syringe pump can accurately control the pressure of the gas supplied by the gas cylinder. $\mathrm{FR}_{22}$ will be controlled directly by $\mathrm{DP}_{22}$ because the temperature controlling system is able to provide heat and maintain the temperature of the foaming chamber. $\mathrm{FR}_{23}$ will be affected by both $\mathrm{DP}_{21}$ and $\mathrm{DP}_{22}$ because gas pressure and temperature influence the concentration of gas that can be dissolved into the plastic sample. $\mathrm{FR}_{23}$ will also depend on $\mathrm{DP}_{23}$ because the high pressure chamber maintains the gas pressure. Finally, $\mathrm{FR}_{24}$ will be affected by all of the DPs in this level because the pressure drop rate is depended on the initial pressure of the chamber $\left(\mathrm{DP}_{21}\right)$, the chamber's temperature $\left(\mathrm{DP}_{22}\right)$, the free volume inside the chamber $\left(\mathrm{DP}_{23}\right)$, and the flow resistance of gas along the gas exit path $\left(D_{24}\right)$. The FRs-DPs relationships are summarize as:

$$
\left[\begin{array}{l}
F R_{21} \\
F R_{22} \\
F R_{23} \\
F R_{24}
\end{array}\right]=\left[\begin{array}{llll}
x & o & o & o \\
o & x & o & o \\
x & x & x & o \\
x & x & x & x
\end{array}\right]\left[\begin{array}{l}
D P_{21} \\
D P_{22} \\
D P_{23} \\
D P_{24}
\end{array}\right]
$$

This design is decoupled and hence it was deemed acceptable based on Axiom 1. Further decompositions 
of FRs and DPs were undertaken to generate a detailed design, but is not shown in this paper.

The information axiom was also applied to improve our design through simplification. For example, standard parts with common sizes were used wherever possible (e.g., nuts, bolts, and o-rings for sealing purposes). The number of different parts was also minimized by using common designs and symmetry (e.g., the chamber is symmetric about a center plane). Tolerance analysis was also done on a few critical dimensions (e.g., the clearances between the drive shafts that actuate the rollers, the rotary seals and the sealing cap that keeps them in place). Based on the analysis, parts were selected or machined in ways to guarantee a high probability of achieving the functional requirements (e.g., to ensure that the clearances mentioned above are within an upper limit beyond which the sealing ability of the rotary seal could be compromised).

Afterward, the foaming system was constructed and tests were performed to verify its capability. It was found that as the plastic sample was heated, it soften and the unsupported region of the sample (see Figure 3a) tend to sag under gravity. It was decided a design revision was needed to change the orientation of the sample (see Figure $3 b$ ) and hence the chamber. Therefore, a new chamber stand was designed and constructed to modify the system's orientation. In addition, the AC motor driving the rollers was replaced with a highly accurate stepper motor to improve the position and speed control, thus resulting in more accurate application of strains and strain rate to the plastic sample. After these design iterations, the system is now working properly and interesting insight on plastic foaming behaviour under extensional stresses are being generated [40]. It is also foreseeable that this system will result in more scientific discovery in the near future.

Figure 3. Sample Orientation a) before; b) after

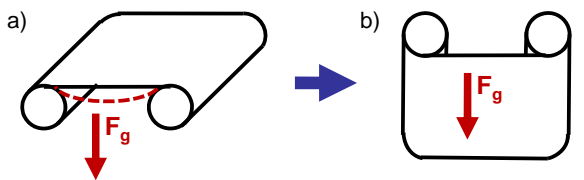

\section{Iterative Design in Education}

As discussed earlier, many researchers have given valuable insight and ideas on improving engineering education in regards to design. This paper aims to add to this research area by discussing possible ideas on course design and assessment/evaluation tools to improve the learning experience of engineering students in design courses. Similar to the view of some other researches, design elements should be taught early and continued throughout the undergraduate engineering curriculum. Iterative behaviour should be encouraged. To this end, more emphasis should be allocated on progress reviews and more marks should be allocated accordingly. In progress reviews, Students should be required to conduct self-critique to encourage reflective thinking, as well as peer-critique to obtain feedback from multiple and diversified perspectives, and to allow students to develop judgement skills in design. For junior engineering students, who might not have extensive design knowledge and experience, design project should be well-defined, so that more time can be spent on implementing iterative design activities. For senior engineering students, design projects should be poor-defined to allow students to further develop their problem scoping skills. Evaluation should be weighted more heavily on progress for junior students to encourage iterative activities, and subsequently shift more weight to final products for senior students. This will better prepare them to solve real-life engineering problems after they graduate, where performances are mostly judged based on outcomes. In all cases, students should be required to self-critique and answer reflective questions such as "If you are to do the project again, what would you have done differently?” in their final reports to foster students' iterative and reflective behaviours. When resources permit, more elaborate feedback should be given to students after completion of projects. However, students often pay little attention to them. Therefore, topics and scope of design projects should be selected carefully to maintain students' interest beyond project completion.

\section{Conclusion}

This paper presented a case study of the iterative design process of a plastic foaming visualization system based on the axiomatic design methodology. Possible ideas to improve engineering curriculum in regards to design teaching were also discussed. By doing so, this paper aims to increase the awareness of the engineering education community regarding the importance of iteration design methodologies.

\section{References}

[1] M.L. Dertouzos, "Made in America: Regaining the productivity edge”, MIT Press, 1989.

[2] B.R. Inman, "Gaining new ground: Technology priorities for America’s future”, Council on Competitiveness, 1991.

[3] J.W. Prados, “Can ABET really make a difference?”, International Journal of Engineering Education, 20, 2004, pp. 315-317. 
[4] E. May, "Is Engineering Education Delivering What Industry Requires?", Paper presented at the $3^{\text {rd }}$ CDEN/RCCI International Design Conference, 2006.

[5] N. National Research Council, Engineering education: Designing an adaptive system, National Academy Press, Washington, 1995.

[6] N. National Science Foundation, Shaping the future: New expectations for undergraduate education in science, mathematics, engineering, and technology, Advisory Committee to the NSF Directorate for Education and Human Resources, Washington, 1996.

[7] N. National Society of Professional Engineers, Engineering education issues: Reporting on surveys of opinions by engineering deans and employers of engineering graduates on the first professional degree, NSPE, Alexandria, 1992.

[8] ABET, Engineering Criteria 2000: Criteria for accrediting programs in engineering in the United States $\left(2^{\text {nd }}\right.$ edition ed.), Engineering Accreditation Commission, Baltimore, 1998.

[9] CEAB, "Accreditation Criteria and Procedures 2009", Canadian Engineering Accreditation Board, 2009, pp. 14.

[10] H.W.J. Rittel, "Some principles for the design of an educational system for design”, Journal of Architectural Education,26(102),1967, pp. 16-26.

[11] N. Cross, A history of design methodology, Kluwer Academic Publishers, The Netherlands, 1992.

[12] D.P. Grant, Housing locations for low income residents: An architectural case study of simulating conflicts of interest and generating compromise proposals, Kluwer Academic Publishers, The Netherlands, 1992.

[13] J. Eekels \& N.F.M. Roonzenburyg, "A methodological comparison of the structures of scientific research and engineering design: Their similarities and differences", Design Studies, 12(4), 1991, pp. 197-203.

[14] J.S. Gero, "Design prototypes: A knowledge representation schema for design”, AI Magazine, 11(4), 1990, pp. 26-36.

[15] D. Braha \& O. Maimon, "The design process: Properties, paradigms, and structures”, IEEE Transactions on Systems, Man, and Cybernetics- Part A: Systems and Humans, 27(2), 1997, pp. 146-166.

[16] I. Hybs \& J.S. Gero, “An evolutionary process model of design”, Design Studies, 13(3), 1992, pp. 273-290.

[17] R.S. Adams, "Cognitive Processes in Iterative Design Behaviour (Thesis)”, PhD Thesis, University of Washington, 2001, pp. 260-261.

[18] E.B. Danielson \& S.G. Danielson, "Problem solving: Improving a critical component of engineering education", Paper presented at the Annual Conference of the American Society of Engineering Education, 1992.

[19] V.G. Cox, "An application of cognitive science to understanding problem solving activity for well structured problems: Cognition, algorithms, metacognition and heuristics”, Paper presented at the Annual Frontiers in Education Conference, 1987.

[20] R. Devon and D. Dorricott, “The pedagogy of design”, Paper presented at the Annual Conference of the American Society of Engineering Education, 1996.
[21] Atman et al., "Teaching engineering design: Can reading a textbook make a difference?”, Research in Engineering Design, 1996, pp. 240-250.

[22] D.F. Radcliffe \& T.Y. Lee, "Design methods used by undergraduate engineering students”, Design Studies, 10(4), 1989, pp. 199-207.

[23] N.P. Suh, The Principles of Design, Oxford University Press, New York, 1990.

[24] N.P. Suh, Axiomatic Design: Advances and Applications, Oxford University Press, New York, 2001.

[25] A.F. Sutcliffe \& N.A.M. Maiden, "Analyzing the novice analyst: Cognitive models in software engineering”, International Journal of Man-Machine Studies, 36, 1992, pp. 719-740.

[26] M.T.H. Chi, M. Bassok, Learning from examples via self-explanations, Lawrence Erlbaum Associates, Hillsdale, 1989.

[27] M.T.H. Chi, N. Leeuw, M.H. Chiu, C. LaVancher, SelfExplanations Improve Learning, 1992.

[28] N. Cross and A.C. Cross, "Observations of teamwork and social processes in design”, Design Studies, 16(2), 1995, pp. 143-170.

[29] D.G. Ullman, T.G. Dietterich \& L.A. Stauffer, “A model of the mechanical design process based on empirical data”, AI EDAM, 2(1), 1988, pp. 33-52.

[30] C.W.J. Ennis and S.W. Gyeszly, "Protocol analysis of the engineering systems design process", Research in Engineering Design, 3, 1991, pp.15-22.

[31] S. Dwarakanath and K.M. Wallace, "Decision-making in engineering design: Observations from design experiment”, Journal of Engineering Design, 6(3), 1995, pp. 191-206.

[32] V. Goel, Sketches of thought, MIT Press, Cambridge, 1995.

[33] D.A. Schön, "Problems, frames, and perspectives on designing”, Design Studies, 5(3), 1984, pp. 133-136.

[34] D.F. Baldwin, D. Tate, C.B. Park, S.W. Cha, N.P. Suh, "Microcellular Plastics Processing Technology", Journal of Japan Society of Polymer Processing, 6, 1994, pp. 245-256.

[35] L.M. Matuana, C.B. Park, J. Balatinecz, "Structures and Mechanical Properties of Microcellular Foamed Polyvinyl Chloride”, Journal of Cellular Plastics, 17, 1998, pp. 1-16.

[36] C.B. Park, D.F. Baldwin, N.P. Suh, "Effect of Pressure Drop Rate on Cell Nucleation in Continuous Processing of Microcellular Polymers," Polymer Engineering and Science, 35(5), 1995, pp. 432-440.

[37] S.T. Lee, "Shear Effects on Thermoplastic Foam Nucleation”, Polymer Engineering Science, 33(7), 1993, pp. 418-422.

[38] L. Chen, H. Sheth, X. Wang, "Effects of Shear Stress and Pressure Drop Rate on Microcellular Foaming Process", Journal of Cellular Plastics, 37, 2001, pp. 353-363.

[39] W. Zhu, N. Zhou, J. Qu, X. Xu, L. Kong, "Effects of Mechanical Vibration on Cell Density and Cell Morphology in the Dynamic Microcellular Foaming Process”, Journal of Cellular Plastics, 42, 2006, pp. 49-61.

[40] A. Wong, R.K.M. Chu, S.N. Leung, C.B. Park, “The Effect of Extensional Stress on Foaming behaviors of Polystyrene Blown with Carbon Dioxide”, SPE ANTEC 2010, Papers \#598, Orlando, Florida, May 16-20, 2010. 